\title{
The use of pioglitazone and the risk of bladder cancer in people with type 2 diabetes: nested case-control study

\author{
Laurent Azoulay assistant professor ${ }^{12}$, Hui Yin statistician ${ }^{1}$, Kristian B Filion assistant professor ${ }^{13}$, \\ Jonathan Assayag graduate student ${ }^{1}$, Agnieszka Majdan endocrinologist ${ }^{4}$, Michael N Pollak \\ oncologist and professor ${ }^{2}$, Samy Suissa professor ${ }^{5}$
}

${ }^{1}$ Centre for Clinical Epidemiology, Lady Davis Institute, Jewish General Hospital, 3755 Côte Sainte-Catherine, H-425.1, Montreal, Quebec, Canada, H3T 1E2; ${ }^{2}$ Department of Oncology, McGill University, Montreal, Quebec, Canada; ${ }^{3}$ Division of Clinical Epidemiology, McGill University, Montreal; ${ }^{4}$ Division of Endocrinology, Jewish General Hospital, Montreal; ${ }^{5}$ Department of Epidemiology, Biostatistics, and Occupational Health, McGill University, Montreal

\begin{abstract}
Objective To determine if the use of pioglitazone is associated with an increased risk of incident bladder cancer in people with type 2 diabetes.

Design Retrospective cohort study using a nested case-control analysis.

Setting Over 600 general practices in the United Kingdom contributing to the general practice research database.

Participants The cohort consisted of people with type 2 diabetes who were newly treated with oral hypoglycaemic agents between 1 January 1988 and 31 December 2009. All incident cases of bladder cancer occurring during follow-up were identified and matched to up to 20 controls on year of birth, year of cohort entry, sex, and duration of follow-up. Exposure was defined as ever use of pioglitazone, along with measures of duration and cumulative dosage.
\end{abstract}

Main outcome measure Risk of incident bladder cancer associated with use of pioglitazone.

Results The cohort included 115727 new users of oral hypoglycaemic agents, with 470 patients diagnosed as having bladder cancer during follow-up (rate 89.4 per 100000 person years). The 376 cases of bladder cancer that were diagnosed beyond one year of follow-up were matched to 6699 controls. Overall, ever use of pioglitazone was associated with an increased rate of bladder cancer (rate ratio 1.83, 95\% confidence interval 1.10 to 3.05 ). The rate increased as a function of duration of use, with the highest rate observed in patients exposed for more than 24 months (1.99, 1.14 to 3.45$)$ and in those with a cumulative dosage greater than $28000 \mathrm{mg}(2.54,1.05$ to 6.14$)$.
Conclusion The use of pioglitazone is associated with an increased risk of incident bladder cancer among people with type 2 diabetes.

\section{Introduction}

The safety of pioglitazone, an oral antidiabetic agent in the thiazolidinedione class, is controversial. Although pioglitazone is effective at reducing glycated haemoglobin $\left(\mathrm{HbA}_{1 \mathrm{c}}\right)$ levels and may decrease the risk of cardiovascular events, ${ }^{12}$ it has also been associated with weight gain and an increased risk of congestive heart failure. ${ }^{1}$ Although available data are limited, there is now some evidence suggesting that pioglitazone may be associated with an increased risk of bladder cancer. ${ }^{3-5}$ In the Prospective Pioglitazone Clinical Trial in Macrovascular Events (PROactive) trial, the reported incidence of bladder cancer was higher among participants randomised to pioglitazone than among those randomised to placebo (14 $v 6$ cases), although this failed to reach statistical significance $(\mathrm{P}=0.069) .{ }^{1}$ However, it was later reported that one case in the placebo group showed benign histology, ${ }^{6}$ and the exclusion of this case resulted in a statistically significant increased risk of bladder cancer (14 $v 5$ cases, relative risk $2.83,95 \%$ confidence interval 1.02 to 7.85 ). ${ }^{7}$ With respect to observational studies, a signal was observed in the US Food and Drug Administration adverse event reporting system. ${ }^{3}$ Furthermore, an interim analysis of an ongoing US cohort did not find an association between pioglitazone and bladder cancer overall but found a $40 \%$ increased risk in patients who used the drug for more than 24 months. ${ }^{4}$ In contrast, a modest increased risk was observed overall in a French cohort. ${ }^{5}$ 
Based on these findings, France decided to suspend the sale of pioglitazone, whereas Germany and Luxembourg recommended that doctors should not start new patients on this drug. ${ }^{8}$ After carrying out a review of the safety of pioglitazone, the European Medicines Agency decided to maintain the marketing authorisation of the drug, ${ }^{8}$ whereas the FDA and Health Canada added warnings of a possible increased risk of bladder cancer in the product monograph. ${ }^{9}{ }^{10}$ Two subsequently published Taiwanese studies found no statistically significant association between pioglitazone and bladder cancer. ${ }^{11} 12$

All of the aforementioned studies included prevalent users of antidiabetic drugs, which may have underestimated the strength of the association between pioglitazone and bladder cancer. $^{4511}{ }^{12}$ As available data on the reported association between pioglitazone and bladder cancer are limited, additional studies are needed to inform regulatory agencies, doctors, and patients on its long term safety. We carried out a population based study to determine if pioglitazone is associated with an increased risk of bladder cancer in people with type 2 diabetes.

\section{Methods}

This study was carried out using the UK general practice research database, the world's largest computerised database of longitudinal records from primary care. ${ }^{13}$ It contains the complete primary care medical record of more than 10 million people enrolled in more than 600 general practices. The geographical distribution of the practices has been shown to be representative of the UK population, and age and sex distributions of patients in the database are similar to those reported by the national population census. ${ }^{14}$ Participating general practitioners have been trained to record medical information, including demographic data, medical diagnoses, procedures, and deaths using a standardised form. Prescriptions issued by doctors using the general practice research database are automatically transcribed into the computer record. In addition, the database collects information on lifestyle variables such as body mass index, and quantitative and qualitative data pertaining to smoking and alcohol use. The Read classification is used to enter medical diagnoses and procedures, and prescriptions are recorded based on the UK Prescription Pricing Authority Dictionary. The recorded information on diagnoses and drug use has been validated and proved to be of high quality. ${ }^{15-18}$

\section{Study population}

Using the general practice research database, we identified all patients who were prescribed their first ever oral antidiabetic agent, with at least one year of medical history in the database before that prescription, between 1 January 1988 and 31 December 2009. The date of entry to the cohort was defined by the date of this first prescription. The antidiabetic agents considered at cohort entry consisted of sulfonylureas (for example, glyburide, gliclazide, glipizide), metformin, thiazolidinediones (pioglitazone, rosiglitazone), and other oral hypoglycaemic agents (meglitinides, dipeptidyl peptidase-4 inhibitors, alpha-glucosidase inhibitors, glucagon-like peptide-1 analogues, and guar gum). We excluded those patients who started treatment with insulin as they were more likely to have type 1 diabetes or advanced type 2 diabetes. Patients who started insulin during follow-up, however, were retained in the cohort. We excluded patients who were less than 40 years of age at the time of their first oral antidiabetic prescription, as well as those with a history of bladder cancer at any time before cohort entry. Participants were followed until a diagnosis of bladder cancer, death from any cause, end of registration with the general practice, or end of the study period (31 December 2009), whichever came first.

\section{Case-control selection}

To investigate the association between pioglitazone and the risk of bladder cancer we carried out a nested case-control analysis. We used this approach because of the time varying nature of drug use, the size of the cohort, and the long duration of follow-up. ${ }^{19}$ Compared with a full cohort approach using a survival analysis with time dependent variables, a nested case-control analysis is computationally more efficient, ${ }^{20}$ while producing odds ratios that are unbiased estimators of incidence rate ratios with little or no loss in precision. ${ }^{19-21}$

We used Read codes to identify all incident cases of bladder cancer occurring during follow-up. For each case, we used the date of the cancer diagnosis to define the index date. Up to 20 controls were randomly selected from the case's risk set, after matching on year of birth, year of cohort entry, sex, and duration of follow-up. By definition, all controls were alive, had no previous diagnosis of bladder cancer, and were registered with their general practice when they were matched to a given case, and thus had equal duration of medical history information at the risk set date. The date of the risk set defined the index date for controls. To consider a latency time window we restricted all analyses to cases and matched controls with at least one year of follow-up between cohort entry and index date.

\section{Use of thiazolidinediones}

For cases and controls we obtained information on prescription of all antidiabetic agents prescribed at any time before the index date. For all definitions of use, including the other antidiabetic agents, we did not consider use initiated in the year immediately before the index date to take into account a biologically meaningful latency time window, and to minimise reverse causation, where initiation of a treatment may have been influenced by early signs or symptoms of cancer.

The definition of primary exposure focused on the ever use of pioglitazone and rosiglitazone, defined by the presence of at least one prescription between cohort entry and the year before the index date. Thus patients were classified into one of four mutually exclusive categories for use of thiazolidinediones: exclusive ever use of pioglitazone, exclusive ever use of rosiglitazone, ever use of both pioglitazone and rosiglitazone (mainly switchers from one drug to the other), and never use of any thiazolidinedione. Never users served as the reference category in the analyses.

To examine potential dose-response relations between pioglitazone and bladder cancer we redefined use in secondary analyses. Therefore, for patients deemed to be exclusive ever users of pioglitazone in the primary exposure definition, we calculated their cumulative duration of use, defined as the time between the first and last prescription, plus the duration of that last prescription. To increase the comparability of our results with those of previous observational studies, ${ }^{45}$ we classified cumulative duration of use as $\leq 12$ months, 13-24 months, and $>24$ months. Finally, in the third approach we computed the cumulative dosage by multiplying the daily dose of each pioglitazone prescription by its specified prescription duration and adding these prescription specific values across all prescriptions received by a given patient between cohort entry and index date. As with cumulative duration, we categorised this variable using the same cut-offs as used in previous 
studies $^{45}: \leq 10500 \mathrm{mg}, 10501-28000 \mathrm{mg}$, and >28 $000 \mathrm{mg}$. Similar dose-response analyses were also done for rosiglitazone.

\section{Statistical analysis}

We used conditional logistic regression to estimate rate ratios and corresponding $95 \%$ confidence intervals for the association between pioglitazone use and the risk of incident bladder cancer. In our first model we evaluated whether exclusive ever use of pioglitazone, when compared with never use of any thiazolidinedione, was associated with an increased risk of bladder cancer. Identical analyses were done for the thiazolidinedione rosiglitazone, which has not been associated with an increased risk of bladder cancer. In a sensitivity analysis to assess the potential impact of confounding by indication, we compared exclusive ever users of pioglitazone with exclusive ever users of rosiglitazone.

In our second and third models we evaluated whether there was a dose-response relation between exclusive ever use of pioglitazone and bladder cancer in terms of cumulative duration of use and cumulative dosage. These analyses were also done for rosiglitazone. We assessed linear trend by entering these dose-response variables as continuous variables in the conditional logistic models. In addition to year of birth, year of cohort entry, sex, and duration of follow-up (on which the logistic regression models were conditioned), we adjusted for several potential confounders measured at any time before cohort entry: $\mathrm{HbA}_{1 \mathrm{c}}$ (last measure before cohort entry), excessive alcohol use (based on alcohol related disorders, such as alcoholism, alcoholic cirrhosis of the liver, alcoholic hepatitis and failure, and other related disorders), obesity (body mass index $\geq 30 \mathrm{~kg} / \mathrm{m}^{2}$ ), smoking (ever versus never), previous cancer (other than non-melanoma skin cancer), previous bladder conditions (such as cystitis and bladder stones), and Charlson comorbidity score, ${ }^{22}$ modified for use in the general practice research database ${ }^{23}$ and adapted not to include diabetes and previous cancer, to avoid duplicate adjustments. Models were further adjusted for ever use of other antidiabetic agents (metformin, sulfonylureas, insulins, and other oral hypoglycaemic agents), defined as at least one prescription between cohort entry and the year before index date. For all models we calculated absolute adjusted rate differences and corresponding $95 \%$ confidence intervals using the formula: $\mathrm{R}_{0}^{*}(1-\mathrm{RR})$, where $\mathrm{R}_{0}$ was the overall bladder cancer rate in the cohort and RR was the adjusted rate ratio for bladder cancer in relation to use of pioglitazone.

We carried out three sensitivity analyses to assess the robustness of our findings. Initially we restricted all analyses to cases and matched controls with at least one year of follow-up and excluded antidiabetic drugs started during the year before the index date to consider a latency time window. However, to account for the possibility that pioglitazone may act as a promoter rather than an inductor of bladder cancer, we repeated the analyses without considering a latency time window. In a second sensitivity analysis we assessed potential

misclassification of use by redefining ever use of pioglitazone and rosiglitazone as receiving at least three prescriptions within a 12 month period, thus minimising the inclusion of patients who may not have been regular users or used these drugs sporadically. Finally, in a third sensitivity analysis we repeated the cumulative duration of use and cumulative dosage analyses by categorising these variables in tertile categories, based on the distribution of use in the controls. All analyses were done with SAS version 9.2 (SAS Institute, Cary, NC).

\section{Results}

Of the 241111 patients prescribed antidiabetic agents during the study period, 115727 met the study inclusion criteria (figure $\Downarrow$ ). The mean age at cohort entry was 64.1 (SD 12.0) years, and the mean duration of follow-up was 4.6 (SD 3.6) years. At cohort entry, the median $\mathrm{HbA}_{\mathrm{lc}}$ was $8.2 \%$, and in descending order, most patients received metformin monotherapy (67\%), followed by sulfonylurea monotherapy (30\%), and thiazolidinedione monotherapy $(0.5 \%)$, whereas the rest used other agents or combinations of agents (3\%). No patients received insulin at cohort entry, as per the inclusion criteria. The mean duration of pioglitazone use among controls was 2.2 (SD 1.5) years (range 28 days to 6.7 years) and was similar for rosiglitazone, where the mean duration was 2.3 (SD $1.5)$ years (range 28 days to 7.6 years).

A total of 470 patients had a diagnosis of incident bladder cancer during 526559 person years of follow-up, yielding an overall rate for bladder cancer of 89.4 per 100000 person years $(95 \%$ confidence interval 81.4 to 97.7); the incidence rate of bladder cancer in the general UK population was 73 per 100000 person years in 2008 , for a population aged at least 65 years. ${ }^{24}$ The higher incidence rate observed in our cohort is consistent with data suggesting an association between type 2 diabetes and an increased risk of bladder cancer. ${ }^{25}$

The primary analyses were restricted to 376 cases, matched to 6699 controls, with at least one year of follow-up between cohort entry and index date to account for latency. Table $1 \Downarrow$ presents the characteristics of these cases and matched controls. Cases were predominantly male. Known risk factors significantly associated with bladder cancer were smoking, previous diagnoses of cancer (other than non-melanoma skin cancer), and a higher comorbidity score. Table $2 \Downarrow$ presents the patterns of use of the different antidiabetic agents, measured between cohort entry and the year before the index date, among cases and matched controls.

Exclusive ever use of pioglitazone was associated with an $83 \%$ increased rate of bladder cancer (adjusted rate ratio 1.83, 95\% confidence interval 1.10 to 3.05 , table $3 \Downarrow$ ). This corresponded to an absolute adjusted rate difference of 74 per 100000 person years (95\% confidence interval 9 to 140). This effect was not observed for exclusive ever use of rosiglitazone (adjusted rate ratio $1.14,95 \%$ confidence interval 0.78 to 1.68 ), the other thiazolidinedione available in the United Kingdom during the study period.

Participants prescribed thiazolidinediones were more likely to be obese, to have ever smoked, and to have uncontrolled diabetes than those who never used any thiazolidinedione. Exclusive ever users of pioglitazone and rosiglitazone, however, were generally similar on most variables, and both groups had lower comorbidity scores than participants who never used any thiazolidinedione (see supplementary table 1). In a sensitivity analysis to assess confounding by indication, we compared exclusive ever users of pioglitazone with exclusive ever users of rosiglitazone. The adjusted rate ratio was numerically increased but did not reach statistical significance (adjusted rate ratio $1.60,95 \%$ confidence interval 0.88 to 2.90 ) owing to few users $(19 v 36)$. None the less, the results of this sensitivity analysis are consistent with those of our primary analysis.

There was evidence of a dose-response relation between pioglitazone use and the rate of bladder cancer (table $4 \Downarrow$ ). In terms of cumulative duration of use, the rate of bladder cancer increased as a function of duration of use, with the highest rate observed in users of more than 24 months (adjusted rate ratio $1.99,95 \%$ confidence interval 1.14 to 3.45 ), corresponding to 
an absolute adjusted rate difference of 88 per 100000 person years (95\% confidence interval 12 to 165 ). For cumulative dosage, a statistically significant association was observed in patients who received more than $28000 \mathrm{mg}(2.54,1.05$ to 6.14$)$, corresponding to an absolute adjusted rate difference of 137 per 100000 person years ( $95 \%$ confidence interval 4 to 271 ). There was no evidence of a dose-response relation with rosiglitazone, both for cumulative duration of use and cumulative dosage (see supplementary table 2).

\section{Sensitivity analyses}

To incorporate a latency time window, the primary analysis was restricted to the 376 cases and their matched controls that had at least one year of follow-up before their index date. In a sensitivity analysis, we repeated the analyses without considering a latency time window. These analyses included all 490 cases and their matched controls. The exclusion of the latency window did not alter our results appreciably, as only one case was exposed to pioglitazone in the first year of follow-up (data not shown). In a second sensitivity analysis, we redefined exclusive ever use of pioglitazone and rosiglitazone as receiving at least three prescriptions within a 12 month period. Compared with the results of the primary analysis, this analysis yielded a slightly higher point estimate for pioglitazone (adjusted rate ratio $1.88,95 \%$ confidence interval 1.10 to 3.22 ) but a slightly lower one for rosiglitazone (1.11, 0.74 to 1.66$)$, indicating minimal misclassification of use. Finally, categorising cumulative duration and cumulative dosage of pioglitazone into three categories produced results consistent with those of our primary analyses on duration and dose (data not shown).

\section{Discussion}

The use of pioglitazone in patients newly treated with oral hypoglycaemic agents is associated with a significant increased risk of bladder cancer. This effect was not observed with rosiglitazone, confirming a drug specific effect. Furthermore, a dose-response relation was observed for cumulative duration of use, with the highest risk observed in participants who used pioglitazone for more than 24 months. A similar dose-response relation was observed in patients who received a high cumulative dosage of pioglitazone. These findings remained consistent in several sensitivity analyses.

\section{Comparison with previous studies}

To our knowledge, few observational studies have examined the association between pioglitazone use and the risk of bladder cancer. ${ }^{3-5} 1112$ The first study did not find an overall increase in risk (hazard ratio $1.2,95 \%$ confidence interval 0.9 to 1.5 ), although a modest increased risk was observed after two years of use $(1.4,1.03$ to 2.0$) .{ }^{4}$ The second study found that pioglitazone was associated with a modest increased risk of bladder cancer overall (hazard ratio 1.22, 95\% confidence interval 1.05 to 1.43 ), which was driven by an increased risk after two years of use $(1.34,1.04$ to 1.79$) .{ }^{5}$ In the third study, ever use of pioglitazone was not associated with an increased risk of bladder cancer overall (odds ratio $0.95,95 \%$ confidence interval 0.70 to 1.29 ), although an increased risk could not be excluded with more than three years of use (1.56, 0.51 to 4.47). In the fourth study, ever use of pioglitazone was not statistically associated with an increased risk of bladder cancer, but the study was possibly underpowered owing to the few cases of bladder cancer included in the analysis $(\mathrm{n}=165) .{ }^{12}$ By combining both new and prevalent users of antidiabetic drugs, these previous studies may have underestimated the association between pioglitazone and bladder cancer. ${ }^{26}$ Specifically, patients receiving pioglitazone who developed bladder cancer before entry to the cohort were, according to the inclusion criteria, selected out of the previous studies. Thus these designs may have missed several cases of bladder cancer that developed among pioglitazone users, a limitation further exacerbated by the fact that this therapy seems to increase the risk over a relatively short duration of use - that is, after two years. Furthermore, the magnitude of the association observed in our study (twofold increased risk after 24 months of use) is consistent with the nearly threefold increased risk observed in the PROactive trial after an average three year follow-up ( 14 cases in the pioglitazone group versus 5 in the placebo group). ${ }^{7}$

\section{Possible biological mechanisms}

Thiazolidinedione peroxisome proliferator activated receptor gamma (PPAR $\gamma$ ) ligands have complex biological effects, ${ }^{27} 28$ but no obvious a priori mechanism links these agents to bladder cancer. In fact, growth inhibitory actions attributed to on-target effects of PPAR ligands have been reported in tissue culture systems, and these results do not predict increased risk of carcinogenesis. ${ }^{29-31}$ However, bladder tumours have been reported in laboratory animals exposed to compounds with PPAR $\gamma$ activity, although these models are of uncertain relevance to human carcinogenesis, as considerable species specificity has been noted, with pioglitazone induced bladder cancer being documented in rats but not in mice. ${ }^{32}$ One line of research implicates chronic bladder irritation as a result of crystal formation, rather than PPAR related signalling, in the observed urothelial carcinogenesis. ${ }^{33}$ It is of interest that muraglitazar, another PPAR agonist that has been experimentally associated with bladder cancer in rats, ${ }^{34}$ is also associated with urolithiasis. However, additional research is needed to elucidate the mechanisms by which a subset of pioglitazone PPAR agonists may increase the risk of bladder cancer, and specifically to determine if risk is indeed correlated with increased urinary crystals in humans, and if so to determine the basis of the crystal formation.

\section{Strengths and limitations of the study}

This population based study has several strengths and some potential limitations. We assembled a large cohort of patients with type 2 diabetes, followed for up to 22 years. Thus the size and long term follow-up of patients enabled the identification of a large number of bladder cancer cases with varying duration of diabetes. In addition, because the general practice research database contains prerecorded information on prescriptions, the possibility of recall bias was eliminated. However, drug information in the general practice research database represents prescriptions written by general practitioners. As such, it is unknown whether prescriptions were actually filled at the pharmacy and whether patients fully adhered to the treatment regimen. Furthermore, prescriptions written by specialists or issued during hospital stay are not recorded in the database. Such misclassification of drug use would be non-differential and therefore bias the results towards the null.

Thiazolidinediones, such as pioglitazone, are indicated for patients intolerant of metformin or sulfonylureas, for patients in whom these drugs are contraindicated, and for patients with poorly controlled diabetes already taking other oral hypoglycaemic agents. Thus people receiving thiazolidinediones are typically at an advanced disease stage than those receiving other oral agents ${ }^{35}$ which may in itself be associated with an increased risk of bladder cancer. ${ }^{36} \mathrm{We}$ attempted to control for this variable by matching cases and controls on duration of 
follow-up (that is, the time since start of oral antidiabetic therapy), which has been shown to be a good proxy for disease severity, ${ }^{37}$ and adjusting the models for $\mathrm{HbA}_{1 \mathrm{c}}$, although this information was missing for $19 \%$ of the cases and controls. However, the lack of any association with rosiglitazone (a thiazolidinedione sharing the same indication as pioglitazone) both in crude and adjusted models and our direct comparison of pioglitazone with rosiglitazone in a sensitivity analysis strongly suggests that disease severity did not confound the association between pioglitazone and bladder cancer. The general practice research database does not collect information on tumour grade and stage but has been shown to have comparable case ascertainment rates as UK cancer registries. ${ }^{38}$ Another limitation of the general practice research database is the lack of information on certain risk factors for bladder cancer. These include exposure to arsenic, occupational exposures, race/ethnicity, and family history of bladder cancer. However, it is unlikely that these variables were differentially distributed between ever users of pioglitazone and ever users of other oral hypoglycaemic agents. Thus we do not believe that the absence of these variables affected the internal validity of the study, although residual confounding may still be present. Finally, the general practice research database contains information on several important confounders, such as body mass index, excessive alcohol use, and smoking. Therefore we were able to adjust for a number of important variables often absent in administrative databases.

\section{Conclusions and policy implications}

In summary, the results of this study provide evidence that pioglitazone is associated with an increased risk of bladder cancer, whereas no increased risk was observed with the thiazolidinedione rosiglitazone. The increased risk associated with pioglitazone became apparent after use for at least 24 months and receiving cumulative dosages greater than 28000 $\mathrm{mg}$. Such associations may have been underestimated in the previous observational studies that included prevalent users. While the magnitudes of the rate ratios were relatively high, the risks associated with pioglitazone are in absolute terms low. Indeed, in this study the highest durations of use and cumulative dosage increased the rate of bladder cancer by 88 and 137 cases per 100000 person years, respectively. Thus doctors, patients, and regulatory agencies should be aware of this association when assessing the overall risks and benefits of this therapy.

LA is the recipient of a Chercheur-Boursier award from the Fonds de la recherche en santé du Québec and SS is the recipient of the James McGill Chair

Contributors: All authors participated in the study design. SS acquired the data. LA and HY did the analyses. LA wrote the manuscript and all authors participated in the interpretation of the results and critical revision of the manuscript. LA is the guarantor.

Funding: This study was supported by grants from the Canadian Institutes of Health Research and the Canadian Foundation for Innovation. The funding sources had no role in the design, analysis, and interpretation of the results, and thus the authors were independent from the funding source.

Competing interests: All authors have completed the ICMJE uniform disclosure form at www.icmje.org/coi_disclosure.pdf (available on request from the corresponding author) and declare: MNP served as a consultant for Novo Nordisk and Sanofi-Aventis and received research funding from Novo Nordisk; no other financial relationships with any organisations that might have an interest in the submitted work in the previous three years; and no other relationships or activities that could appear to have influenced the submitted work.
Ethical approval: This study was approved by the independent scientific advisory committee of the general practice research database and the research ethics committee of the Jewish General Hospital, Montreal, Canada.

Data sharing: No additional data available.

1 Dormandy JA, Charbonnel B, Eckland DJ, Erdmann E, Massi-Benedetti M, Moules IK, et al. Secondary prevention of macrovascular events in patients with type 2 diabetes in the PROactive Study (PROspective pioglitAzone Clinical Trial In macroVascular Events): a randomised controlled trial. Lancet 2005;366:1279-89.

2 Lincoff AM, Wolski K, Nicholls SJ, Nissen SE. Pioglitazone and risk of cardiovascular events in patients with type 2 diabetes mellitus: a meta-analysis of randomized trials. JAMA 2007;298:1180-8.

3 Piccinni C, Motola D, Marchesini G, Poluzzi E. Assessing the association of pioglitazone use and bladder cancer through drug adverse event reporting. Diabetes Care 2011;34:1369-71.

4 Lewis JD, Ferrara A, Peng T, Hedderson M, Bilker WB, Quesenberry CP Jr, et al. Risk of bladder cancer among diabetic patients treated with pioglitazone: interim report of a longitudinal cohort study. Diabetes Care 2011;34:916-22.

5 Caisse nationale de l'assurance maladie. Risque de cancer de la vessie chez les personnes diabétiques traitées par pioglitazone en France: une étude de cohorte sur les données du SNIIRAM et du PMSI. Agence française de sécurité sanitaire des produits de santé. 2011. www.afssaps.fr/var/afssaps_site/storage/original/application/ b42a6bf9a1b63c3dbec7388d3914687b.pdf.

6 Dormandy J, Bhattacharya M, van Troostenburg de Bruyn AR. Safety and tolerability of pioglitazone in high-risk patients with type 2 diabetes: an overview of data from PROactive. Drug Saf 2009;32:187-202.

7 Hillaire-Buys D, Faillie JL, Montastruc JL. Pioglitazone and bladder cancer. Lancet 2011;378:1543-4.

8 Questions and answers on the review of pioglitazone-containing medicines (Actos, Glustin, Competact, Glubrava and Tandemact). European Medicines Agency. 2011. www.ema. europa.eu/docs/en_GB/document_library/Medicine_QA/2011/07/WC500109179.pdf.

9 Food and Drug Administration. FDA Drug Safety Communication: update to ongoing safety review of Actos (pioglitazone) and increased risk of bladder cancer. FDA Drug Safety Communication. 2011. www.fda.gov/Drugs/DrugSafety/ucm259150.htm\#aihp.

10 Health Canada. Health Canada reviewing diabetes drug pioglitazone (Actos) and potential risk of bladder cancer. Health Canada. 2011. www.hc-sc.gc.ca/ahc-asc/media/advisoriesavis/_2011/2011_79-eng.php.

11 Chang CH, Lin JW, Wu LC, Lai MS, Chuang LM, Chan KA. Association of thiazolidinediones with liver cancer and colorectal cancer in type 2 diabetes mellitus. Hepatology 2011;55:1462-72.

12 Tseng $\mathrm{CH}$. Pioglitazone and bladder cancer: a population-based study of Taiwanese. Diabetes Care 2012;35:278-80.

13 Walley T, Mantgani A. The UK General Practice Research Database. Lancet 1997;350:1097-9.

14 Garcia Rodriguez LA, Perez GS. Use of the UK General Practice Research Database for pharmacoepidemiology. Br J Clin Pharmacol 1998;45:419-25.

15 Jick $\mathrm{H}$, Jick SS, Derby LE. Validation of information recorded on general practitioner based computerised data resource in the United Kingdom. BMJ 1991;302:766-8.

16 Lawrenson R, Williams T, Farmer R. Clinical information for research; the use of general practice databases. J Public Health Med 1999;21:299-304.

17 Lawrenson R, Todd JC, Leydon GM, Williams TJ, Farmer RD. Validation of the diagnosis of venous thromboembolism in general practice database studies. Br J Clin Pharmacol 2000;49:591-6.

18 Jick SS, Kaye JA, Vasilakis-Scaramozza C, Garcia Rodriguez LA, Ruigomez A, Meier $\mathrm{CR}$, et al. Validity of the general practice research database. Pharmacotherapy 2003;23:686-9.

19 Suissa S. Novel approaches to pharmacoepidemiology study design and statistical analysis. In: Strom B, ed. Pharmacoepidemiology. 4th ed. Wiley; 2005:811-29.

20 Essebag V, Platt RW, Abrahamowicz M, Pilote L. Comparison of nested case-control and survival analysis methodologies for analysis of time-dependent exposure. BMC Med Res Methodol 2005;5:5.

21 Essebag V, Genest J Jr, Suissa S, Pilote L. The nested case-control study in cardiology. Am Heart J 2003;146:581-90.

22 Charlson ME, Pompei P, Ales KL, MacKenzie CR. A new method of classifying prognostic comorbidity in longitudinal studies: development and validation. $J$ Chronic Dis 1987:40:373-83.

23 Khan NF, Perera R, Harper S, Rose PW. Adaptation and validation of the Charlson Index for Read/OXMIS coded databases. BMC Fam Pract 2010;11:1.

24 Cancer Research UK. Bladder cancer (C67), average number of new cases per year and age-specific incidence rates, UK, 2006-2008. Cancer Research UK. 2012. http://info. cancerresearchuk.org/cancerstats/types/bladder/incidence/.

25 Larsson SC, Orsini N, Brismar K, Wolk A. Diabetes mellitus and risk of bladder cancer: a meta-analysis. Diabetologia 2006;49:2819-23.

26 Ray WA. Evaluating medication effects outside of clinical trials: new-user designs. Am J Epidemiol 2003;158:915-20.

27 Kahn BB, McGraw TE. Rosiglitazone, PPARgamma, and type 2 diabetes. N Engl J Med 2010;363:2667-9.

28 Choi JH, Banks AS, Kamenecka TM, Busby SA, Chalmers MJ, Kumar N, et al. Antidiabetic actions of a non-agonist PPARgamma ligand blocking Cdk5-mediated phosphorylation. Nature 2011;477:477-81

29 Yoshimura R, Matsuyama M, Segawa Y, Hase T, Mitsuhashi M, Tsuchida K, et al. Expression of peroxisome proliferator-activated receptors (PPARs) in human urinary bladder carcinoma and growth inhibition by its agonists. Int J Cancer 2003;104:597-602.

30 Nakashiro KI, Hayashi Y, Kita A, Tamatani T, Chlenski A, Usuda N, et al. Role of peroxisome proliferator-activated receptor gamma and its ligands in non-neoplastic and neoplastic human urothelial cells. Am J Pathol 2001;159:591-7.

31 Guan YF, Zhang YH, Breyer RM, Davis L, Breyer MD. Expression of peroxisome proliferator-activated receptor gamma (PPARgamma) in human transitional bladder cancer and its role in inducing cell death. Neoplasia 1999;1:330-9. 


\section{What is already known on this topic}

The association between use of the thiazolidinedione antidiabetic agent pioglitazone and bladder cancer is controversial The few population based studies on this subject produced conflicting results

Additional studies are needed to confirm or refute this association

\section{What this study adds}

Overall, the use of pioglitazone, and not the thiazolidinedione rosiglitazone, was associated with an increased risk of bladder cancer This risk increased with duration of use and cumulative dosage and was highest among those using the drug for more than 24 months and those receiving cumulative dosages greater than $28000 \mathrm{mg}$

32 Suzuki S, Arnold LL, Pennington KL, Kakiuchi-Kiyota S, Wei M, Wanibuchi H, et al. Effects of pioglitazone, a peroxisome proliferator-activated receptor gamma agonist, on the urine and urothelium of the rat. Toxicol Sci 2010;113:349-57.

33 Sato K, Awasaki Y, Kandori H, Tanakamaru ZY, Nagai H, Baron D, et al. Suppressive effects of acid-forming diet against the tumorigenic potential of pioglitazone hydrochloride in the urinary bladder of male rats. Toxicol Appl Pharmacol 2011;251:234-44.

34 Dominick MA, White MR, Sanderson TP, Van VT, Cohen SM, Arnold LE, et al. Urothelial carcinogenesis in the urinary bladder of male rats treated with muraglitazar, a PPAR alpha/gamma agonist: evidence for urolithiasis as the inciting event in the mode of action. Toxicol Pathol 2006;34:903-20.

35 Edwards KL, Alvarez C, Irons BK, Fields J. Third-line agent selection for patients with type 2 diabetes mellitus uncontrolled with sulfonylureas and metformin. Pharmacotherapy 2008;28:506-21.

36 MacKenzie T, Zens MS, Ferrara A, Schned A, Karagas MR. Diabetes and risk of bladder cancer: evidence from a case-control study in New England. Cancer 2011;117:1552-6.
37 Benoit SR, Fleming R, Philis-Tsimikas A, Ji M. Predictors of glycemic control among patients with type 2 diabetes: a longitudinal study. BMC Public Health 2005;5:36.

38 Van Staa TP, Patel D, Gallagher AM, de Bruin ML. Glucose-lowering agents and the patterns of risk for cancer: a study with the General Practice Research Database and secondary care data. Diabetologia 2012;55:654-65.

Accepted: 18 April 2012

\section{Cite this as: BMJ 2012;344:e3645}

This is an open-access article distributed under the terms of the Creative Commons Attribution Non-commercial License, which permits use, distribution, and reproduction in any medium, provided the original work is properly cited, the use is non commercial and is otherwise in compliance with the license. See: http://creativecommons.org/licenses/bync/2.0/ and http://creativecommons.org/licenses/by-nc/2.0/legalcode. 


\section{Tables}

Table 1| Baseline characteristics of bladder cancer cases and matched controls. Values are numbers (percentages) unless stated otherwise

\begin{tabular}{|c|c|c|c|}
\hline Characteristics & Cases $(n=376)$ & Controls ( $n=6699$ ) & Crude rate ratio $(95 \% \mathrm{Cl})$ \\
\hline Mean $(\mathrm{SD})$ age $(\text { years })^{*}$ & $68.9(9.3)$ & $68.9(8.6)$ & - \\
\hline Men $^{*}$ & $306(81.4)$ & $5400(81.4)$ & - \\
\hline Mean (SD) duration of follow-up (years) ${ }^{*}$ & $4.8(3.2)$ & $4.8(2.7)$ & - \\
\hline Excessive alcohol use $†$ & $23(6.1)$ & $430(6.4)$ & $0.95(0.63$ to 1.45$)$ \\
\hline \multicolumn{4}{|l|}{ Body mass index $\left(\mathrm{kg} / \mathrm{m}^{2}\right)$ : } \\
\hline$<30$ & $222(59.0)$ & $4171(62.3)$ & 1.00 (reference) \\
\hline$\geq 30$ & $140(37.2)$ & $2416(36.1)$ & $1.08(0.88$ to 1.34$)$ \\
\hline Unknown & $14(3.7)$ & $112(1.7)$ & 2.20 (1.28 to 3.78$)$ \\
\hline \multicolumn{4}{|l|}{ Smoking status: } \\
\hline Never & $102(27.1)$ & $2743(41.0)$ & 1.00 (reference) \\
\hline Ever & $240(63.8)$ & $3361(50.2)$ & $1.86(1.47$ to 2.34$)$ \\
\hline Unknown & $34(9.0)$ & $595(8.9)$ & 1.51 (1.02 to 2.22$)$ \\
\hline \multicolumn{4}{|l|}{$\mathrm{HbA}_{10}:$} \\
\hline$\leq 7.4 \%$ & $198(52.7)$ & $3384(50.5)$ & 1.00 (reference) \\
\hline$>7.4 \%$ & $106(28.2)$ & $2042(30.5)$ & 0.89 (0.71 to 1.13$)$ \\
\hline Unknown & $72(19.2)$ & $1273(19.0)$ & $0.97(0.74$ to 1.27$)$ \\
\hline Previous bladder conditions & $23(6.1)$ & $335(5.0)$ & $1.28(0.82$ to 2.01$)$ \\
\hline Previous cancerł & $50(13.3)$ & $481(7.2)$ & 1.89 (1.40 to 2.55$)$ \\
\hline Mean (SD) Charlson comorbidity score§ & $1.07(1.34)$ & $0.95(1.17)$ & $1.10(1.01$ to 1.20$)$ \\
\hline
\end{tabular}

*Matching variables (along with year of cohort entry). For controls, means and percentages were weighted by the inverse number of controls matched to each case.

†Based on alcohol related disorders, such as alcoholism, alcoholic cirrhosis of the liver, alcoholic hepatitis and failure, and other related disorders. $\ddagger$ Cancers other than non-melanoma skin cancer.

$\S$ Calculation based on presence of 19 categories of comorbidity. 
Table 2| Patterns of antidiabetic agents received between cohort entry and year before index date among cases of bladder cancer and matched controls*

\begin{tabular}{|c|c|c|c|}
\hline Antidiabetic agents & No $(\%)$ of cases $(n=376)$ & No $(\%)$ of controls $(n=6699)$ & Crude rate ratio $(95 \% \mathrm{Cl})$ \\
\hline Metformin only & $119(31.7)$ & $2104(31.4)$ & 1.00 (reference) \\
\hline Sulfonylureas only & $69(18.4)$ & $1564(23.4)$ & 0.62 (0.44 to 0.87$)$ \\
\hline Metformin and sulfonylureas & $99(26.3)$ & $1688(25.2)$ & $0.98(0.72$ to 1.33$)$ \\
\hline Metformin and thiazolidinediones & $12(3.2)$ & $231(3.5)$ & $0.99(0.53$ to 1.86$)$ \\
\hline Metformin, sulfonylureas, and thiazolidinediones & $23(6.1)$ & $381(5.7)$ & $1.06(0.64$ to 1.75$)$ \\
\hline Metformin, sulfonylureas, and insulin & $15(4.0)$ & $211(3.2)$ & $1.04(0.56$ to 1.91$)$ \\
\hline Metformin, sulfonylureas, and other agents $\dagger$ & $7(1.9)$ & $72(1.1)$ & $1.49(0.65$ to 3.44$)$ \\
\hline $\begin{array}{l}\text { Metformin, sulfonylureas, thiazolidinediones, and } \\
\text { insulin }\end{array}$ & $9(2.4)$ & $80(1.2)$ & 1.86 (0.86 to 4.02$)$ \\
\hline Other treatment combinations & $23(6.1)$ & $368(5.5)$ & $1.03(0.63$ to 1.68$)$ \\
\hline
\end{tabular}

*Matched on year of birth, year of cohort entry, sex, and duration of follow-up.

†This category included meglitinides, dipeptidyl peptidase-4 inhibitors, alpha-glucosidase inhibitors, glucagon-like peptide-1 analogues, and guar gum. These categories are mutually exclusive. 
Table 3 | Thiazolidinediones and risk of bladder cancer among cases of bladder cancer and matched controls*

\begin{tabular}{|c|c|c|c|c|}
\hline Use of thiazolidinediones & No $(\%)$ of cases $(n=376)$ & No $(\%)$ of controls $(n=6699)$ & Crude rate ratio $(95 \% \mathrm{Cl})$ & Adjusted rate ratio $(95 \% \mathrm{Cl}) \dagger$ \\
\hline Never use of any thiazolidinedione & $319(84.8)$ & $5856(87.4)$ & 1.00 (reference) & 1.00 (Reference) \\
\hline Exclusive ever use of pioglitazone & $19(5.1)$ & $191(2.9)$ & 1.87 (1.13 to 3.09$)$ & 1.83 (1.10 to 3.05$)$ \\
\hline Exclusive ever use of rosiglitazone & $36(9.6)$ & $596(8.9)$ & 1.16 (0.79 to 1.69$)$ & 1.14 (0.78 to 1.68$)$ \\
\hline $\begin{array}{l}\text { Ever use of both pioglitazone and } \\
\text { rosiglitazone }\end{array}$ & $2(0.5)$ & $56(0.8)$ & $0.74(0.18$ to 3.08$)$ & $0.78(0.18$ to 3.29$)$ \\
\hline
\end{tabular}
rosiglitazone

*Matched on year of birth, year of cohort entry, sex, and duration of follow-up.

†Adjusted for excessive alcohol use, obesity, smoking status, $\mathrm{HbA}_{1 \mathrm{c}}$, previous bladder conditions, previous cancer (other than non-melanoma skin cancer), Charlson comorbidity score, and ever use of other antidiabetic agents (metformin, sulfonylureas, insulin, and other oral hypoglycaemic agents). 
Table 4| Pioglitazone cumulative duration of use and cumulative dosage and risk of bladder cancer among cases of bladder cancer and matched controls*

\begin{tabular}{|c|c|c|c|c|}
\hline Variables & No $(\%)$ of cases $(n=376)$ & $\begin{array}{c}\text { No }(\%) \text { of controls } \\
(n=6699)\end{array}$ & Crude rate ratio $(95 \% \mathrm{Cl})$ & Adjusted rate ratio $(95 \% \mathrm{Cl}) \dagger$ \\
\hline Never use of any thiazolidinediones & $319(84.8)$ & $5856(87.4)$ & 1.00 (reference) & 1.00 (reference) \\
\hline \multicolumn{5}{|l|}{$\begin{array}{l}\text { Cumulative duration of } \\
\text { pioglitazone: }\end{array}$} \\
\hline$\leq 12$ months & $1(0.3)$ & $27(0.4)$ & $0.69(0.09$ to 5.11$)$ & $0.56(0.07$ to 4.42$)$ \\
\hline 13-24 months & $2(0.5)$ & $11(0.2)$ & $2.99(0.61$ to 14.59$)$ & 3.03 (0.63 to 14.52$)$ \\
\hline \multirow[t]{2}{*}{$>24$ months } & $16(4.3)$ & $153(2.3)$ & 2.00 (1.16 to 3.45$)$ & 1.99 (1.14 to 3.45$)$ \\
\hline & & & & $P=0.050$ for trend \\
\hline \multicolumn{5}{|l|}{ Cumulative dosage of pioglitazone: } \\
\hline$\leq 10500 \mathrm{mg}$ & $7(1.9)$ & $70(1.0)$ & $1.63(0.72$ to 3.69$)$ & 1.58 (0.69 to 3.62$)$ \\
\hline $10501-28000 \mathrm{mg}$ & $6(1.6)$ & $68(1.0)$ & $1.75(0.75$ to 4.07$)$ & $1.66(0.70$ to 3.94$)$ \\
\hline \multirow[t]{2}{*}{$>28000 \mathrm{mg}$} & $6(1.6)$ & $53(0.8)$ & 2.44 (1.02 to 5.84$)$ & 2.54 (1.05 to 6.14$)$ \\
\hline & & & & $P=0.030$ for trend \\
\hline
\end{tabular}

*Matched on year of birth, year of cohort entry, sex, and duration of follow-up.

†Adjusted for excessive alcohol use, obesity, smoking status, $\mathrm{HbA}_{10}$, previous bladder conditions, previous cancer (other than non-melanoma skin cancer), Charlson comorbidity score, and ever use of other antidiabetic agents (metformin, sulfonylureas, insulin, and other oral hypoglycaemic agents). 


\section{Figure}

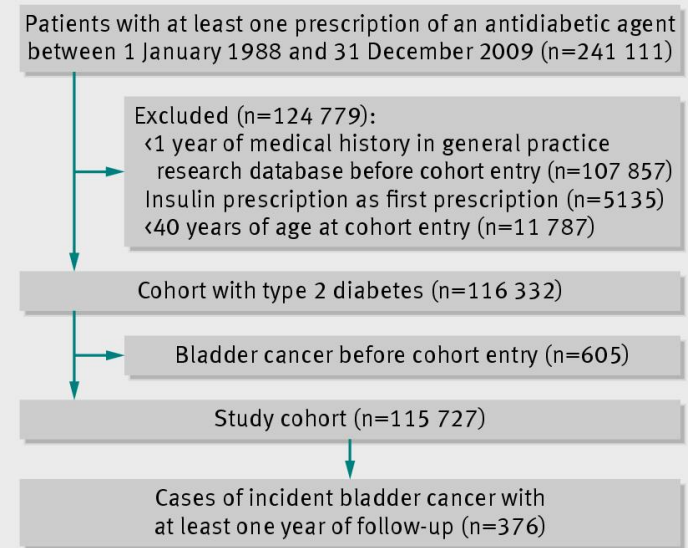

Fig 1 Flow of participants through study 